British Journal of Psychiatry (1995), 167, 692-700

\title{
Correspondence
}

Contents: A 'PC' model of the mind/Lead-in placebo washout period/Obstetric complications in schizophrenia/Eugenics/Mania precipitated by carbamazepine withdrawal/Laryngeal dystonia/Risperidone response after no clozapine response

\section{A 'PC' model of the mind}

SIR: Charlton (1995) presents a radical cognitive neuropsychiatric model of the mind and its disorders. The commentators make several points, and others come to mind.

(a) While syndromal diagnoses have many limitations, to discard them would be to throw the baby out with the bathwater. The distinction between their clinical worth and their explanatory power (made by David in his commentary) is critical. Clinically, it is largely a matter of convenience whether particular symptoms and signs are grouped together as a syndrome. For explanatory purposes, it is a matter of fact whether a given syndrome corresponds meaningfully to one aetiopathogenic entity, to several, or to none. Within the syndrome of dementia, for example, there are clinically and pathologically recognisable individual disorders, but the concept of dementia remains. Moreover, the individual dementia disorders have a variable relationship with aetiological and pathogenic mechanisms: one disorder can have multiple causes, such as Alzheimer's disease; conversely, one gene can cause distinct clinical syndromes, e.g. the prion diseases. There is no single rule, perhaps no rule at all, governing the relationship between a genetic cause, pathogenic mechanisms (including neuropsychological processes), and clinical syndrome; neither is there any absolute truth about what constitutes a 'disease'. Certainly other psychiatric syndromes such as schizophrenia may prove to be replaceable both by more useful and by more valid categories; on the other hand they may not. It is premature to advocate the demise of syndromes qua syndromes, or to conclude that any other explanatory level is inherently more likely to carve mental illness at whatever joints it has. These are all empirical facts waiting to be determined. (b) It is unfair to select out biological psychiatry for criticism on the grounds that it will not find a cause for a syndrome if the syndrome doesn't exist. Any approach based on syndromal classification is subject to the same vulnerability, be it molecular, psychological or social. In any case, appropriately designed biological studies can investigate subsyndromal or symptom-level features. Demonstration of blood flow changes associated with poverty of speech independent of diagnosis exemplify this (Dolan et al, 1993).

(c) Charlton's model not only has the major difficulties of any Cartesian theory of mind - who would argue for a computer literate homunculus? but it is unclear if the "brain-is-a-computer" parallel is being drawn as a metaphor or as a serious view of how it works (Soyland, 1994). If it is the former, the metaphor is being taken too far; if the latter, it is as unproven as every previous explanation of the mind-brain which called upon contemporary technologies of the era. What is the evidence for the strict separation of the software from the operating system, or from the hardware? Or that psychiatric disorders exclusively affect the operating system? A central theme of neurobiology is that the brain has a dynamic synaptic, chemical and metabolic organisation which allows no firm distinction of structure from function. Neither do such models give due weight to the brain abnormalities seen in schizophrenia and other syndromes which, while far from being sufficient, mean that the computer itself and its hardware malfunctions are a necessary element in any explanatory model, whether of a syndrome, a symptom, or a cognitive module.

Charlton, B. G. (1995) Cognitive neuropsychiatry and the future of diagnosis: a 'PC' model of the mind. British Journal of Psychiatry, 167, 149-158.

Dolan, R. J., Bench, C. J., Liddle, P. F., et al (1993) Dorsolateral prefrontal cortex dysfunction in the major psychoses: Symptom or disease specificity. Journal of Neurology, Neurosurgery and Psychiatry, 56, 1290-1294.

Sorland, A. J. (1994) Psychology as Metaphor. London: Sage.

P. J. HARRISON

J. GEDDES

Warneford Hospital

Oxford OX3 7JX 